\title{
Influence of Camembert consumption on the composition and metabolism of intestinal microbiota: a study in human microbiota-associated rats
}

\author{
Christophe Lay ${ }^{1}$, Malène Sutren ${ }^{1}$, Pascale Lepercq ${ }^{1}$, Catherine Juste ${ }^{1}$, Lionel Rigottier-Gois ${ }^{1}$, \\ Evelyne Lhoste ${ }^{1}$, Riwanon Lemée ${ }^{2}$, Pascale Le Ruyet ${ }^{2}$, Joël Doré ${ }^{1}$ and Claude Andrieux ${ }^{1} *$ \\ ${ }^{1}$ UEPSD, Centre de Recherche de Jouy en Josas, INRA, 78352 Jouy en Josas Cedex, France \\ ${ }^{2}$ Lactalis Recherche et Développement, 53089 Laval Cedex, France
}

(Received 3 November 2003 - Revised 25 February 2004 - Accepted 30 March 2004)

\begin{abstract}
The objective of the present study was to evaluate the consequence of Camembert consumption on the composition and metabolism of human intestinal microbiota. Camembert cheese was compared with milk fermented by yoghurt starters and Lactobacillus casei as a probiotic reference. The experimental model was the human microbiota-associated (HM) rat. HM rats were fed a basal diet (HMB group), a diet containing Camembert made from pasteurised milk (HMCp group) or a diet containing fermented milk (HMfm group). The level of micro-organisms from dairy products was measured in faeces using cultures on a specific medium and PCR-temporal temperature gradient gel electrophoresis. The metabolic characteristics of the caecal microbiota were also studied: $\mathrm{SCFA}, \mathrm{NH}_{3}$, glycosidase and reductase activities, and bile acid degradations. The results showed that micro-organisms from cheese comprised $10^{5}-10^{8}$ bacteria/g faecal sample in the HMCp group. Lactobacillus species from fermented milk were detected in HMfm rats. Consumption of cheese and fermented milk led to similar changes in bacterial metabolism: a decrease in azoreductase activity and $\mathrm{NH}_{3}$ concentration and an increase in mucolytic activities. However, specific changes were observed: in HMCp rats, the proportion of ursodeoxycholic resulting from chenodeoxycholic epimerisation was higher; in HMfm rats, $\alpha$ and $\beta$-galactosidases were higher than in other groups and both azoreductases and nitrate reductases were lower. The results show that, as for fermented milk, Camembert consumption did not greatly modify the microbiota profile or its major metabolic activities. Ingested micro-organisms were able to survive in part during intestinal transit. These dairy products exert a potentially beneficial influence on intestinal metabolism.
\end{abstract}

Cheese: Camembert: Human microbiota-associated rats: Intestinal microbiota: Probiotics

There is growing evidence that the balance of gastrointestinal microbiota and microbiota functions play an important role in maintaining health and preventing diseases. The colonic microbiota is a complex ecosystem, which until recently was identified using cultures of specific medium and phenotypic characteristics. During the last 10 years, molecular methods have revealed that $70-80 \%$ of micro-organisms escape analysis by bacteriological culture methods (Suau et al. 1999). The molecular techniques based on the detection of nucleotide sequence of $16 \mathrm{~S}$ rRNA and the amplification of 16S rDNA sequences, coupled with separation by denaturing gel electrophoresis, allow a better assessment of the microbiota composition and dominant species complexity (Blaut et al. 2002).

Functional foods contain ingredients that beneficially affect the health of consumers (Salminen et al. 1998). Among these functional foods, yoghurt and fermented milks provide live bacteria called probiotics, belonging mainly to Lactobacillus and Bifidobacterium species, although some other species or other micro-organisms, such as yeast, have been a matter of interest (De Roos \& Katan, 2000; Heyman \& Ménard, 2002). In human subjects, several studies have shown that these micro-organisms resist endogenous digestion during intestinal transit and are found at high levels in the stools during fermented milk consumption (Marteau et al. 1994; Bezkorovainy, 2001). There is also some evidence that probiotics improve the intestinal metabolism, modulate immunity and prevent intestinal diseases such as diarrhoea (Goldin, 1998; Marteau et al. 2002). However, experimental studies suggest that each strain and each association of strains lead to specific effects (Sanders, 1993; Reid, 1999).

One of the possible mechanisms of action of probiotics is an effect on the composition and the metabolism of the intestinal microbiota. In children, we have previously shown that fermented milk containing Lactobacillus casei DN-114 001 and yoghurt symbiosis have positive effects on diarrhoea and regulate some deleterious bacterial activities, such as reductase and $\beta$-glucuronidase (Guérin-Danan et al. 1998). Similar observations have been obtained in

\footnotetext{
Abbreviations: HM, human microbiota-associated; HMB, group fed on basal diet; HMCp, group fed on diet containing Camembert made from pasteurised milk; HMfm, group fed on diet containing fermented milk; Psc, Pearson similarity coefficient; TTGE, temporal temperature gradient gel electrophoresis. * Corresponding author: Dr Claude Andrieux, fax+33134652492, email andrieux@jouy.inra.fr
} 
human microbiota-associated (HM) rats (Djouzi et al. 1997).

In an effort to expand the products providing probiotics, food such as Cheddar cheese, Gouda cheese, cottage cheese or ice-cream have been studied as food-carriers of both Bifidobacterium and Lactobacillus probiotic strains (Stanton et al. 1998). However, nothing is known about the effect of flowered-rind soft cheese containing live bacteria such as Camembert, which is traditionally consumed in France and many other countries worldwide (Lenoir et al. 1995).

The objective of the present study was to evaluate the consequence of Camembert on intestinal microbiota composition and metabolism. The model used is the HM rat, as described by Djouzi et al. (1997). The influence of Camembert consumption on dominant intestinal microbiota was evaluated using PCR-temporal temperature gradient electrophoresis (TTGE) analysis. The concentration of common cheese micro-organisms was measured in faecal samples using both cultures on specific medium and PCR-TTGE analysis with specific primers. The metabolic characteristics of the faecal microbiota were studied: SCFA as markers for glycolytic fermentation, $\mathrm{NH}_{3}$ and iso-acids as markers for proteolytic fermentation, and glycosidases involved in carbohydrate hydrolysis $(\alpha$ - and $\beta$-galactosidase, $\alpha$ - and $\beta$-glucosidase) and in mucin degradation (neuraminidase, $\beta$ - $N$-acetyl-galactosaminidase and $\alpha$-Lfucosidase). The $\beta$-glucuronidase, nitrate reductase and azoreductase activities and bile acid conversions leading to the release of toxic substances were also investigated. Milk fermented by yoghurt starters and Lactobacillus case $i$ was used as a probiotic-containing reference product.

\section{Methods}

\section{Animals and diets}

Germ-free male Fischer rats (2.5 months old, $n$ 30; UEPSD, Jouy en Josas, France) were reared in three Texler-type isolators (La Cahlène, Vélizy, France). The rats were fed the basal diet $B$ (Table 1) for 1 week. They were then inoculated through a single oral gavage with

Table 1. Composition of the diets

\begin{tabular}{lrrr}
\hline Diet... & B & Cp & $\mathrm{fm}$ \\
\hline Basal diet components (g/kg) & & & \\
$\quad$ Casein & 50 & 0 & 50 \\
Soyabean-protein isolate & 120 & 120 & 120 \\
Maize starch & 280 & 280 & 280 \\
Mashed potato & 280 & 280 & 280 \\
Sucrose & 40 & 40 & 40 \\
Maize oil & 30 & 30 & 30 \\
Lard & 50 & 0 & 50 \\
Cellulose & 70 & 70 & 70 \\
Mineral and vitamins & 80 & 80 & 80 \\
Diet preparation (g/d per rat) & & & \\
Basal diet & 15 & 15 & 15 \\
Cheese* & 0 & 50 & 0 \\
Fermented milk & 0 & 0 & 50 \\
Water & 20 & 20 & 15 \\
\hline
\end{tabular}

* Protein $200 \mathrm{~g} / \mathrm{kg}$; fat $220 \mathrm{~g} / \mathrm{kg}$.
$1 \mathrm{ml}$ of a $10^{-2}$ dilution of a faecal sample from a 30 year-old healthy female volunteer, who had not received antibiotics for at least 3 months before stool collection. The faecal dilution was performed from a frozen $10^{-1}$ dilution of the faecal sample $\left(-80^{\circ} \mathrm{C}\right.$ into glycerol) in an anaerobic cabinet $\left(\mathrm{N}_{2}-\mathrm{H}_{2}-\mathrm{CO}_{2}(85: 10: 5\right.$, by vol. $\left.)\right)$ in $(\mathrm{g} /$ 1): $\mathrm{NaCl} 5 \cdot 0$, glucose $2 \cdot 0$, cysteine hydrochloride $0 \cdot 3$. For the following 3 weeks, rats were maintained on the basal diet. At $t_{0}$, rats received the $\mathrm{Cp}$ diet (Table 1) containing Camembert made from pasteurised milk (HMCp rats), the fm diet (Table 1) containing fermented milk (HMfm rats) or were kept on the basal diet B (HMB rats) for 6 weeks. The basal diets was sterilised by $\gamma$-irradiation at $45 \mathrm{kGy}$ in plastic vacuum bags and offered as a paste prepared daily (described in Table 1).

The microbial compositions of Camembert and fermented milk are shown in Tables 2 and 3. They were conditioned in sterile pots sealed with a double cover. The pots were placed daily in the isolators using peracetic acid $(100 \mathrm{ml} / \mathrm{l})$.

\section{Sample collection}

Several samples of faeces were collected from the rectum of each individually identified rat before $\left(t_{0}\right)$, at 3 weeks $\left(t_{3}\right)$ and 6 weeks $\left(t_{6}\right)$ under the three dietary conditions. Fresh faecal samples collected at $t_{6}$ were pooled for enumeration of bacteria of dairy-product origin. The other samples were frozen at $-80^{\circ} \mathrm{C}$ until analyses. For molecular analyses, faecal samples from three rats (no. 1, no. 2, no. 3) of each dietary group collected at $t_{0}, t_{3}$ and $t_{6}$ were used individually and in pools. For steroid analysis, faeces were collected at $t_{6}$ over two consecutive $24 \mathrm{~h}$ periods from each cage and stored at $-80^{\circ} \mathrm{C}$ until analysis. The two sequential $24 \mathrm{~h}$ samples were pooled to give a single sample per cage (five rats per dietary group).

Rats were killed using $\mathrm{CO}_{2}$ and their caeca were removed and weighed. The caecal contents were immediately frozen in liquid $\mathrm{N}_{2}$ and stored at $-80^{\circ} \mathrm{C}$ until determinations of enzymic activities and metabolites. The caecal samples from the three rats, no. 1 , no. 2 and no. 3 of each group, were used for PCR-TTGE analysis.

All procedures were conducted in accordance with the Institute's Guide for the Care and Use of Laboratory Animals.

\section{Micro-organism enumeration}

The dairy products were provided from regular factory products every 2 weeks during the experiment. They were maintained at $4{ }^{\circ} \mathrm{C}$ and introduced in isolators three times per week. Microbiological analyses were performed twice on each of the three batches of products: at the beginning and at the end of their use (after 2 weeks storage at $4^{\circ} \mathrm{C}$ ). The crust and the inside of Camembert were analysed separately. Pimaricine ( $25 \mathrm{~g} / \mathrm{l}$ water; $1 \mathrm{ml} / 1$ medium) (Sigma-Aldrich, Saint Quentin Fallavier, France) was added to analyse the crust of cheese in order to eliminate fungal micro-organisms.

The dairy products were analysed using culture in specific medium. Samples were diluted from $10^{-1}$ to 
Table 2. Microbiological analyses (log colony-forming units/g) of Camembert and of faecal samples*

\begin{tabular}{|c|c|c|c|}
\hline Micro-organism & Camembert† & Faeces HMCpł & Faeces $\mathrm{HMB} \ddagger$ \\
\hline Streptococcus thermophilus & $7 \cdot 8-8 \cdot 2$ & $7 \cdot 5$ & ND \\
\hline Lactococcus & $8 \cdot 1-9 \cdot 1$ & ND & ND \\
\hline Leuconostoc mesenteroides & $6 \cdot 0-7 \cdot 5$ & ND & $7 \cdot 5$ \\
\hline Thermophilic Lactobacillus & $0-4$ & ND & ND \\
\hline Mesophilic Lactobacillus & $0-7 \cdot 3$ & $7 \cdot 7$ & ND \\
\hline Hafnia alvei & $4 \cdot 7-8 \cdot 6$ & ND & ND \\
\hline Corynebacteria & $0-5 \cdot 1$ & $6 \cdot 1$ & ND \\
\hline Micrococcaceae & $2 \cdot 7-6 \cdot 8$ & $6 \cdot 8$ & ND \\
\hline Geotrichum & $7.7-7.9$ & $7 \cdot 0$ & ND \\
\hline Yeast & $3 \cdot 7-6 \cdot 8$ & $5 \cdot 3$ & ND \\
\hline Penicillium & $5 \cdot 5-6 \cdot 5$ & ND & ND \\
\hline Enterobacteria & ND & $6 \cdot 8$ & $5 \cdot 75$ \\
\hline Enterococcus & ND & $7 \cdot 5$ & $9 \cdot 0$ \\
\hline
\end{tabular}

Table 3. Microbiological analyses (log colony forming units $/ g$ ) of fermented milk and of faecal samples*

\begin{tabular}{|c|c|c|c|}
\hline Micro-organism & Fermented milk $†$ & Faeces HMfm $\ddagger$ & Faeces HMB‡ \\
\hline Streptococcus thermophilus & $8 \cdot 8-8 \cdot 3$ & ND & ND \\
\hline Lactobacillus casei & $8 \cdot 2-8.5$ & $8 \cdot 3$ & ND \\
\hline Lactobacillus delbruecki bulgaricus & $6 \cdot 3-7 \cdot 1$ & ND & ND \\
\hline
\end{tabular}

$10^{-9}$ in medium containing tryptone $(1 \mathrm{~g} / \mathrm{l}$; Biokar, Beauvais, France) and $\mathrm{NaCl}(9 \mathrm{~g} / \mathrm{l}$; Merck, Nogent sur Maine, France) and then surface-plated $(0.1 \mathrm{ml})$ in duplicate on selective agars.

For lactic acid bacteria, De Mann-Rugosa-Sharpe medium (Difco, Becton Dickinson, Le Pont de Clair, France) was incubated at $30^{\circ} \mathrm{C}$ (mesophile) or $45^{\circ} \mathrm{C}$ (thermophile) for $48 \mathrm{~h}$ in anaerobiosis (Anaerocult; Merck). For Streptococcus thermophilus, M17 medium (Biokar) was used and plates were incubated at $45^{\circ} \mathrm{C}$ for $48 \mathrm{~h}$ in aerobiosis. For Leuconostoc, medium containing tomato juice (Difco) with $5 \mathrm{~g}$ calcium citrate/l (Merck) and $6 \mathrm{mg}$ vancomycine/l (Sigma) was incubated for $3 \mathrm{~d}$ at $22^{\circ} \mathrm{C}$. Enterococci were enumerated after $24 \mathrm{~h}$ incubation at $37^{\circ} \mathrm{C}$ using citrate azide Tween carbonate (Merck).

Micrococcaceae, Enterobacteriaceae and Coryneform bacteria were determined using tryptone soyabean agar (Oxoid, Dardilly, France) with and without $\mathrm{NaCl}(30 \mathrm{~g} / \mathrm{l})$. Bacteria were enumerated after $3 \mathrm{~d}$ incubation at $30^{\circ} \mathrm{C}$.

Geotrichum and yeast were enumerated using modified Czapeck medium (g/l): $\left(\mathrm{NH}_{4}\right)_{2} \mathrm{SO}_{4}$ (Panreac, Lyons, France) 2, $\mathrm{KH}_{2} \mathrm{PO}_{4}$ (Merck) 1; $\mathrm{KCl}$ (Merck) 0.5, $\mathrm{MgSO}_{4} \cdot 7 \mathrm{H}_{2} \mathrm{O}$ (Merck) $0 \cdot 5, \mathrm{FeSO}_{4} \cdot 7 \mathrm{H}_{2} \mathrm{O}$ (VWR, Fontenay sous Bois, France) 0.01, glucose (Merck) 10, yeast extract (Difco) 2, agar (Biokar) 20. Plates were incubated for $6 \mathrm{~d}$ at $25^{\circ} \mathrm{C}$. For Penicillium analysis, medium contained $(\mathrm{g} / \mathrm{l})$ : glucose (Merck) 30, NaCl (Merck) 80, $\mathrm{NaNO}_{3}$ (Merck) 3, $\mathrm{KCl}$ (Merck) 0.5, $\mathrm{MgSO}_{4} \cdot 7 \mathrm{H}_{2} \mathrm{O}$ (Merck) 0.5, $\mathrm{K}_{2} \mathrm{HPO}_{4}$
(Merck) 1, $\mathrm{FeSO}_{4}$ (VWR) 0.01, yeast extract 5, agar (Biokar) 20. Plates were incubated for $6 \mathrm{~d}$ at $25^{\circ} \mathrm{C}$.

Faecal samples were analysed at the end of the experiment using pools from each group. Because the objective was to analyse the microbiota from the dairy products ingested, nalidixic acid ( $30 \mathrm{~g} / \mathrm{l}$; Sigma) and pimaricine ( $25 \mathrm{~g} / \mathrm{l}$ water, $1 \mathrm{ml} / \mathrm{l}$ medium; Sigma), were added to inhibit Gram-negative bacteria.

Gram staining, catalase production and API ${ }^{\circledR}$ plates were also used (BioMérieux, Marcy l'Etoile, France) to improve bacterial identification.

\section{DNA isolation}

Total DNA was extracted from $0.2 \mathrm{~g}$ faecal samples, $1.5 \mathrm{~g}$ cheese or $2 \mathrm{ml}$ fermented milk (pellet from centrifugation at $15000 \mathrm{~g}$ for $15 \mathrm{~min}$ ) in $2.2 \mathrm{ml} \mathrm{screw}$-capped tubes (Sarstedt International, Orsay, France), as described previously (Godon et al. 1997). The concentration and integrity of the nucleic acids were determined visually by electrophoresis on agarose $(10 \mathrm{~g} / \mathrm{l})$ gel containing ethidium bromide.

\section{PCR amplification}

Primers U968-GC (5'-CGC CCG GGG CGC GCC CCG GGC GGG GCG GGG GCA CGG GGG GAA CGC GAA GAA CCT TAC-3') and L1401 (5'-GCG TGT GTA CAA GAC $\mathrm{CC}-3^{\prime}$ ) were used to amplify the V6 to 
V8 regions of the bacterial $16 \mathrm{~S}$ rDNA (Zoetendal et al. 1998). Primers Lac1 or Lac 352-f (5'-AGC AGT AGG GAA TCT TCC A-3') and Lac 2 GC or Lac 679-GC-r (5'-CGC CCG CCG CGC GCG CGG CGG GCG GGG GCG GGG GCG GGG CAC GGG GGG ATT YCA CCG CTA CAC ATG-3') (Walter et al. 2001) were used for specific amplification of the 16S rDNA sequences of Lactobacillus, Pediococcus, Leuconostoc and Weissella genera, belonging to the Lactobacilli group. These two sets of primers produced approximatively 433 and $327 \mathrm{bp}$ PCR amplicons respectively. All primers were purchased from Qbiogene Research Services (Evry, France).

PCR was performed using HotStar Taq DNA polymerase (Qiagen, Courtaboeuf, France). The PCR mix (50 $\mu \mathrm{l})$ contained $1 \times \mathrm{PCR}$ buffer, $1 \mathrm{mM}-\mathrm{MgCl}_{2}, 200 \mu \mathrm{M}$ each dNTP, 20 pmol each primer, 2.5 U Hot Star Taq polymerase and approximately $2 \mathrm{ng}$ DNA. The samples were amplified in a PCT 100 thermocycler (MJ Research, Inc., Albertville, MN, USA) using the following program: $95^{\circ} \mathrm{C}$ for $15 \mathrm{~min}$, thirty cycles of $97^{\circ} \mathrm{C}$ for $1 \mathrm{~min}, 58^{\circ} \mathrm{C}$ for $1 \mathrm{~min}$ and $72^{\circ} \mathrm{C}$ for $90 \mathrm{~s}$, and finally $72^{\circ} \mathrm{C}$ for $15 \mathrm{~min}$ for the first set of primers. Hybridation temperature was $61^{\circ} \mathrm{C}$ for $1 \mathrm{~min}$ for the primers Lac1 and Lac 2GC. PCR products were analysed by electrophoresis on agarose $(10 \mathrm{~g} / \mathrm{l})$ gel containing ethidium bromide to check their size and estimate their concentration.

\section{Temporal temperature gradient gel electrophoresis}

PCR amplification allowed generation of DNA fragments that were of the same size, but variable in sequence. TTGE allows separation of the various DNA sequences as discrete bands by migration in a denaturing polyacrylamide gel containing urea, with a concomitant increase in temperature during electrophoresis.

The DCode Universal Mutation Detection System (Bio-Rad, Paris, France) was used for sequence-specific separation of PCR products. Electrophoresis was performed, as previously described (Seksik et al. 2003), through a $1 \mathrm{~mm}$ thick, $160 \times 160 \mathrm{~mm}$ polyacrylamide $(80 \mathrm{~g} / \mathrm{l})$ gel-acrylamide/Bis (37.5:1.0, v/v), $7 \mathrm{M}$-urea, $1.25 \times$ Tris-acetate-EDTA, and $55 \mu \mathrm{l}$ and $550 \mu \mathrm{l}$ TEMED (Eurobio, Les Ulis, France) and ammonium persulfate $(100 \mathrm{~g} / \mathrm{l})$ respectively using 7 litres $1.25 \times$ Tris acetate-EDTA as the electrophoresis buffer.

At the beginning of electrophoresis, for better resolution the voltage was fixed at $20 \mathrm{~V}$ for $15 \mathrm{~min}$. For the first set of amplicons, electrophoresis was run for $16 \mathrm{~h}$ at $64 \mathrm{~V}$ with an initial temperature of $66^{\circ} \mathrm{C}$ and a ramp rate of $0.2^{\circ} \mathrm{C}$ per $\mathrm{h}$. For amplicons obtained with primers Lac1 and Lac 2GC, the temperature range was $63 \cdot 8-70 \cdot 0^{\circ} \mathrm{C}$ with ramp rate of $0.4^{\circ} \mathrm{C}$ per $\mathrm{h}$ and at $66 \mathrm{~V}$.

Each well was loaded with 100-200 ng amplified DNA plus an equal volume of $2 \times$ gel loading dye (Bromophenol Blue $(0.5 \mathrm{~g} / \mathrm{l})-x y l e n e$ cyanol $(0.5 \mathrm{~g} / \mathrm{l})-$ glycerol $(700 \mathrm{~g} / \mathrm{l}))$. For each gel, three lanes were loaded with a marker obtained by mixing PCR products of seven rDNA clones isolated from one molecular inventory of one human faecal sample (Suau et al. 1999). Clones were related to members of the Clostridium coccoides group, Clostridium leptum subgroup, Bacteroides group and Bifidobacterium group. After the completion of electrophoresis, the gels were stained in the dark by immersion for $30 \mathrm{~min}$ in a solution of SYBR Green I Nucleic Acid Gel Stain (Roche Diagnostics, Meylan, France) and read on a Storm system (Molecular Dynamics, Amersham Biosciences, Orsay, France).

\section{Biochemical analyses}

Enzyme activities were measured in caecal samples using a thermo-regulated anaerobic chamber $\left(\mathrm{H}_{2}-\mathrm{CO}_{2}-\mathrm{N}_{2}\right.$ (10:10:80, by vol.)), as previously described (Andrieux et al. 2002). Samples were diluted 1:20 using pre-reduced PBS (pH 6.7). $\alpha$ - and $\beta$-galactosidase, $\alpha$ - and $\beta$-glucosidase, $\beta$-glucuronidase, $\beta$ - $N$-acetyl-galactosaminidase and $\alpha$-L-fucosidase activities were measured by determining the rate of $p$-nitrophenol released from $p$-nitrophenyl glycosides. Azoreductase activity was determined using amaranth (5 mM) as substrate. Neuraminidase activity was measured using 4-methylumbelliferyl- $N$-acetylneuraminic acid as substrate. Nitrate reductase was determined by the generation of nitrite. Enzyme activities were expressed as $\mu$ mol metabolised substrate per min and per $g$ protein.

Protein concentration was determined in triplicate by the method of Lowry et al. (1951) on 1:500 faecal dilution in $\mathrm{Na}_{2} \mathrm{CO}_{3}(20 \mathrm{~g} / \mathrm{l})$ and $\mathrm{NaOH}(0 \cdot 1 \mathrm{M})$. Bovine serum albumin was used as the standard.

SCFA concentrations in caecal samples were analysed in duplicate after water extraction of acidified samples using GC (Perkin-Elmer 1020 GC; Saint Quentin, France) (Andrieux et al. 2002). $\mathrm{NH}_{3}$ was determined using the Berthelot method adapted by Dropsy \& Boy (1961).

For bile acid analysis in faeces, $4 \mathrm{~g}$ faeces was thoroughly homogenised in a minimal volume of distilled water; lipids were extracted with $100 \mathrm{ml}$ ethanol under reflux for $48 \mathrm{~h}$ in a Soxhlet apparatus. Ethanolic extracts $(4 \mathrm{ml})$ were then saponified $\left(120^{\circ} \mathrm{C}, 3 \mathrm{~h}\right.$, under pressure) in the presence of $1 \mathrm{ml} 10 \mathrm{M}-\mathrm{NaOH}$. Neutral steroids were removed from the saponified mixture, with $3 \times 10 \mathrm{ml}$ light petroleum $\left(40-60^{\circ} \mathrm{C}\right)$ and bile acids were extracted from the remaining acidified $(\mathrm{pH} 2)$ aqueous phase with $3 \times 10 \mathrm{ml}$ diethyl ether. After evaporation of the solvent, bile acids were redissolved in $2 \mathrm{ml}$ methanol. For chromatographic analysis, free bile acids were methylated with diazomethane, then sylilated with $\mathrm{N}, \mathrm{O}$-bis(trimethylsilyl)trifluoroacetamide-triethylchlorosilane and trimethylsilylimidazole-pyridine (1:4, v/v) (Supelco, Saint Quentin Fallavier, France) in dichloromethane. Trimethylsilyl derivates were analysed using a GC Peri 2000 (Perichrom, Saulx-les-Chartreux, France) equipped with a Ross injector and an OV-1 column $(30 \mathrm{~m} \times 0.32 \mu \mathrm{m} \times 0.2 \mu \mathrm{m}$; Perichrom). Respective proportions of the different molecular species of bile acids were determined using calibration lines obtained from the analysis of standard mixtures and $5 \alpha$-cholestan as external standard.

\section{Statistical analyses}

PCR-TTGE profiles were compared using GelCompar software (GelComparII ${ }^{\mathrm{rM}}$, version 2.0; Applied Maths, Kortrigk, Belgium). Analyses take into account number, 
intensity and position of PCR-TTGE bands (PCR-amplified 16S rDNA fragments) in the gel. Pearson correlation provided similarity based upon densitometric curves. Similarity coefficients of Pearson were calculated to generate a similarity matrix. The dendrogram was obtained from this matrix by using the algorithm UPGMA (Unweighted Pair Group Method using Arithmetic averages).

Results of biochemical analyses were expressed as mean values with their standard errors of the mean. Data were analysed using ANOVA (StatView; Abacus Concepts, Berkeley, CA, USA). Where ANOVA indicated significant treatment effects, mean values were compared using the Newman-Keuls procedure. Statistical significance was accepted at $P<0 \cdot 05$.

\section{Results}

Bacterial PCR-temporal temperature gradient gel electrophoresis analysis of faecal samples

At $t_{0}, 3$ weeks after the human microbiota inoculation and before the dairy products were given, faecal samples of three rats per group were analysed individually and in pools. The PCR-TTGE profiles of faecal pools were representative of the profile of individual samples within the pool (Pearson similarity coefficient (Psc) >94\%) (Fig. 1). Faecal microbiota profiles were similar between the three experimental groups (Psc $>95.9 \%$ ).

In the control group (HMB), the comparison between PCR-TTGE profiles of pools of faecal samples at different times ( 3 weeks $\left(t_{0}\right), 6$ weeks $\left(t_{3}\right)$ and 9 weeks $\left(t_{6}\right)$ after human faecal inoculation) indicated a high stability of the microbiota over time for the period of 9 weeks.

As in the control group, in HMCp and HMfm rats fed the dairy products the profiles of faecal pools were representative of the individual profile, over time (Psc $>94$ to $96 \%$ ). In HMCp rats, however, profiles were quite similar at $t_{0}$ and $t_{3}$ (Psc $>93 \%$ ), some bands appeared or disappeared during Camembert consumption (Fig. 2). No differences were observed between profiles obtained at $t_{3}$ and $t_{6}$ (Psc $>95 \%$ )

In HMfm rats, Psc was only $90 \%$ between $t_{0}$ and $t_{3}$ and $96 \%$ between $t_{3}$ and $t_{6}$ (Fig. 3 ).

No bands specific to the PCR-TTGE profiles of dairy products were observed in the profiles of $\mathrm{HMfm}$ and HMCp rats during the experimental diet when bacterial PCR primers were used (Figs 2 and 3).
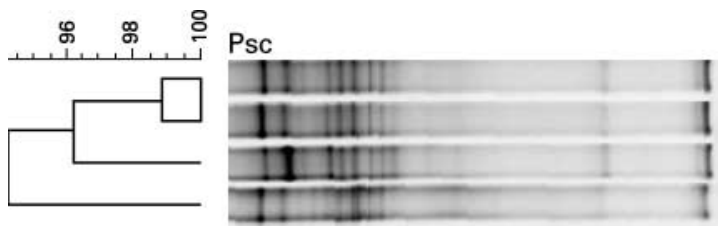

Fig. 1. Comparison between temporal temperature gradient gel electrophoresis profiles of PCR products of the V6 to V8 regions from DNA of pooled and individual faecal samples from human microbiota-associated (HM) rats. (A), faecal sample from $\mathrm{HM}$ rat no. 1 at $t_{0} ;(\mathrm{B})$, faecal sample from HM rat no. 2 at $t_{0} ;(\mathrm{C})$, faecal sample from $\mathrm{HM}$ rat no. 3 at $t_{0}$; (D), pool of faeces from $\mathrm{HM}$ rats no. 1, no. 2 and no. 3 at $t_{0}$. For details of procedures, see p. 430 .

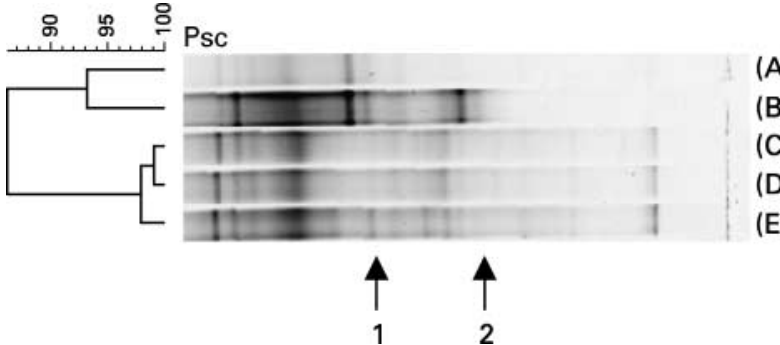

Fig. 2. Comparison between temporal temperature gradient gel electrophoresis profiles of PCR products of the V6 to V8 regions from DNA of the Camembert and faecal samples from a group of human microbiota-associated rats fed on diet containing Camembert made from pasteurised milk (HMCp). (A), Camembert (inside); (B), Camembert (crust); (C), pool of faeces (HMCp group, $n 3$ ) at 6 weeks $\left(t_{6}\right)$; (D), pool of faeces (HMCp group, $n 3$ ) at 3 weeks $\left(t_{3}\right)$; (E) pool of faeces (HMCp group, $n 3$ ) at 0 weeks $\left(t_{0}\right)$. For details of diets and procedures, see Table 1 and p. 430. Alteration of the profile during Camembert consumption is illustrated by arrow 1 showing the disappearance of $a$ band at $t_{3}$ and $t_{6}$ and by arrow 2 showing the appearance of a new band at $t_{3}$ and $t_{6}$.

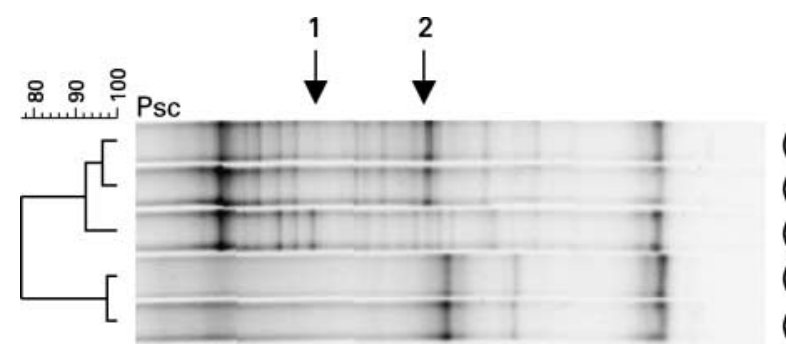

Fig. 3. Comparison between temporal temperature gradient ge electrophoresis profiles of PCR products of the V6 to V8 regions from DNA of the fermented milk and faecal samples from a group of human microbiota-associated rats fed on diet containing fermented milk (HMfm group). (A), pool of faeces (HMfm group, $n 3$ ) at 3 weeks $\left(t_{3}\right)$; $(\mathrm{B})$, pool of faeces (HMfm group, $n 3$ ) at 6 weeks $\left(t_{6}\right)$; (C), pool of faeces (HMfm group, $n 3$ ) at 0 weeks $\left(t_{0}\right)$; (D), fermented milk (one sample); (E), fermented milk (other sample). For details of diets and procedures, see Table 1 and p. 430. Alteration of the profile during fermented milk consumption is illustrated by arrow 1 showing the disappearance of a band at $t_{3}$ and $t_{6}$ and by arrow 2 showing the appearance of a new band at $t_{3}$ and $t_{6}$.

At the end of the experiment, caecal and faecal PCRTTGE profiles were similar in all groups (Psc $>99 \%$ ).

\section{Lactobacilli group-specific PCR-temporal temperature} gradient gel electrophoresis analysis of faecal samples

With Lactobacilli group-specific PCR primers, specific bands from fermented milk were obtained in faecal profiles of HMfm rats (Fig. 4). No bands were obtained for the cheese sample. However, in faecal samples from HMCp rats, Lactobacilli group-specific bands were obtained at $t_{6}$, whereas no band was found at $t_{0}$.

\section{Enumeration in faecal samples of micro-organisms from} Camembert and fermented milk

Results obtained in cheese samples and in faecal samples are reported in Table 2. Camembert contained mainly lactic acid bacteria Lactococcus (8.1-9.1 log colony forming units/g), 


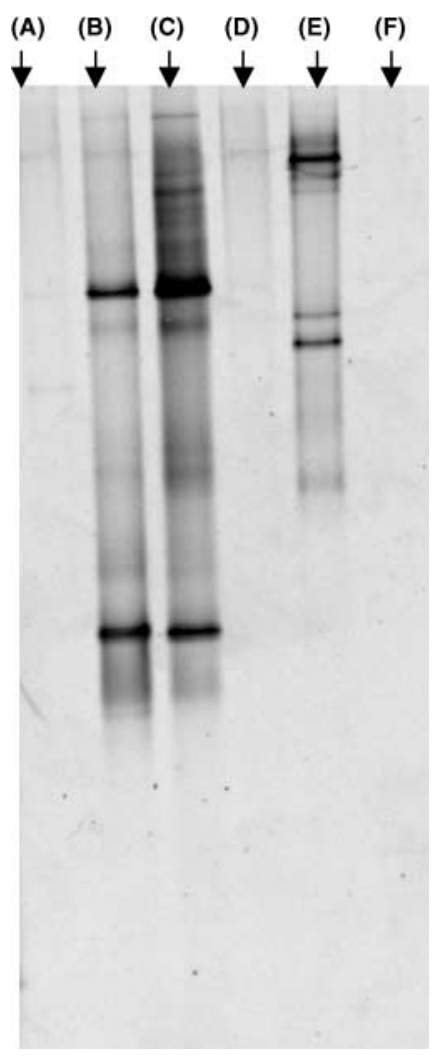

Fig. 4. Temporal temperature gradient gel electrophoresis profiles of Lactobacilli group-specific PCR product from DNA of the fermented milk, Camembert and faecal samples from human microbiotaassociated rats fed diets containing fermented milk ( $\mathrm{HMfm})$ or Camembert (HMCp) at $t_{0}$ (before ingestion of dairy products) and after 6 weeks consumption of dairy products $\left(t_{6}\right)$. (A), HMfm sample at $t_{0} ;(\mathrm{B}), \mathrm{HMfm}$ sample at $t_{6} ;(\mathrm{C})$, fermented milk; (D), HMCp at $t_{0}$; $(\mathrm{E}), \mathrm{HMCp}$ at $t_{6} ;(\mathrm{F})$, Camembert.

Streptococcus thermophilus $(7 \cdot 8-8 \cdot 2 \log$ colony forming units/g), Leuconostoc (6.0-7.5 log colony forming units/ g). Non-starter mesophilic and thermophilic Lactobacillus were found occasionally $(0 \cdot 0-7 \cdot 3 \mathrm{log}$ colony forming units/g). The ripening microbiota was mainly composed of associated Hafnia alvei, coryneform bacteria, Micrococcaceae, Geotrichum, Penicillium and yeast.
Several bacterial species from cheese Streptococcus thermophilus, mesophilic Lactobacillus, Micrococcaceae and Geotrichum were found at levels of 5.3-7.7 log colony forming units/g faecal samples of HMCp rats feeding on Camembert for 6 weeks. Lactococcus, Leuconostoc, Penicillium and Hafnia alvei were not detected (Table 2).

Fermented milk contained high levels of Streptococcus thermophilus, Lactobacillus casei and Lactobacillus delbrueckii bulgaricus (Table 3).

In faecal samples of rats fed the fermented milk for 6 weeks, Lactobacillus casei was found at $8.3 \log$ colony forming units/g, whereas Streptococcus thermophilus and Lactobacillus delbrueckii bulgaricus were not detected (Table 3).

\section{Caecal bacterial metabolism}

Comparison between rats fed the basal diet (HMB) and rats fed Camembert (HMCp) revealed that in caecal samples of rats fed Camembert, azoreductase activity was significantly lower and mucolytic activities (neuraminidase, $N$-acetylgalactosaminidase and fucosidase) were higher (Table 4). SCFA caecal concentration and SCFA profile were not significantly modified, but $\mathrm{NH}_{3}$ caecal concentration was lowered (Table 5).

In caecal samples from the HMfm rats fed fermented milk, $\alpha$ and $\beta$-galactosidase were higher than in other groups. Neuraminidase and fucosidase activities were higher than in the HMB rats, but $N$-acetyl galactosaminidase was not modified. Azoreductase and nitrate reductase activities were both reduced (Table 4). $\mathrm{NH}_{3}$ concentration was lower in HMCp and HMfm rats compared with HMB rats, without change in SCFA profile (Table 5).

\section{Faecal bile acids}

As illustrated in Table 6, only ursodeoxycholic and $\omega$-muricholic acids, resulting from chenodeoxycholic and $\beta$-muricholic microbial epimerisation respectively were significantly affected by diet. When expressed as \% composition of bile acids in faeces, ursodeoxycholic acid, which was not detected in faeces from control rats

Table 4. Enzymic activities ( $\mu \mathrm{mol} / \mathrm{min}$ per g protein) in caecal contents of human microbiotaassociated rats*

(Mean values with their standard errors for ten rats per group)

\begin{tabular}{|c|c|c|c|c|c|c|}
\hline \multirow[t]{2}{*}{ Group... } & \multicolumn{2}{|c|}{ HMB } & \multicolumn{2}{|c|}{ HMCp } & \multicolumn{2}{|c|}{$\mathrm{HMfm}$} \\
\hline & Mean & SEM & Mean & SEM & Mean & SEM \\
\hline$\beta$-Galactosidase & $1.54^{\mathrm{b}}$ & 0.14 & $1 \cdot 50^{\mathrm{b}}$ & 0.17 & $2 \cdot 00^{\mathrm{a}}$ & 0.10 \\
\hline$\alpha$-Galactosidase & $0.66^{\mathrm{b}}$ & 0.03 & $0.63^{b}$ & 0.04 & $0.78^{a}$ & 0.04 \\
\hline$\beta$-Glucosidase & 0.26 & 0.04 & 0.17 & 0.03 & 0.26 & 0.03 \\
\hline$\beta$-Glucuronidase & 0.22 & 0.01 & 0.20 & 0.02 & 0.27 & 0.02 \\
\hline Neuraminidase & $0.05^{b}$ & 0.01 & $0 \cdot 12^{\mathrm{a}}$ & 0.03 & $0 \cdot 10^{\mathrm{a}}$ & 0.02 \\
\hline$N$-acetylgalactosaminidase & $0.061^{b}$ & 0.008 & $0.098^{a}$ & 0.006 & $0.063^{b}$ & 0.005 \\
\hline$\alpha$-L-Fucosidase & $0.058^{b}$ & 0.005 & $0.071^{a b}$ & 0.005 & $0.097^{a}$ & 0.041 \\
\hline Nitrate reductase & $0.083^{a}$ & 0.020 & $0.090^{\mathrm{a}}$ & 0.020 & $0.024^{b}$ & 0.006 \\
\hline Azoreductase & $0.065^{a}$ & 0.010 & $0.025^{\mathrm{b}}$ & 0.008 & $0.024^{b}$ & 0.003 \\
\hline
\end{tabular}

${ }^{a, b}$ Mean values within a row with unlike superscript letters were significantly different $(P<0.05)$.

${ }^{*}$ Rats were fed the basal diet (HMB group), the diet containing Camembert (HMCp group) or fermented milk (HMfm group) for 6 weeks; for details of diets and procedures, see Table 1 and p. 430. 
Table 5. Concentrations of SCFA ( $\mu \mathrm{mol} / \mathrm{g}$ caecal contents), lactic acid and ammonia in caecal contents of human microbiota-associated rats*

(Mean values with their standard errors for ten rats per group)

\begin{tabular}{|c|c|c|c|c|c|c|}
\hline \multirow[t]{2}{*}{ Group... } & \multicolumn{2}{|c|}{$\mathrm{HMB}$} & \multicolumn{2}{|c|}{ HMCp } & \multicolumn{2}{|c|}{ HMfm } \\
\hline & Mean & SEM & Mean & SEM & Mean & SEM \\
\hline SCFA & $76 \cdot 1$ & $7 \cdot 2$ & $69 \cdot 1$ & $3 \cdot 0$ & $64 \cdot 1$ & $7 \cdot 1$ \\
\hline Acetate & $52 \cdot 9$ & 4.8 & $49 \cdot 1$ & $2 \cdot 3$ & $45 \cdot 9$ & $5 \cdot 0$ \\
\hline Propionate & $12 \cdot 6$ & 1.5 & $9 \cdot 3$ & 0.4 & $8 \cdot 8$ & $1 \cdot 0$ \\
\hline Butyrate & 8.4 & 0.9 & 8.5 & 0.4 & $7 \cdot 2$ & 0.9 \\
\hline Valerate + caproate & 1.56 & 0.12 & 1.36 & 0.30 & 1.66 & 0.15 \\
\hline Iso-acids & 0.62 & 0.40 & 0.83 & 0.22 & 0.89 & 0.13 \\
\hline Lactic acid & 4.75 & 0.17 & 3.35 & $0 \cdot 10$ & 3.74 & 0.15 \\
\hline $\mathrm{NH}_{3}$ & $3 \cdot 25^{a}$ & 0.23 & $2.08^{\mathrm{b}}$ & 0.08 & $2 \cdot 28^{\mathrm{b}}$ & 0.22 \\
\hline
\end{tabular}

${ }^{\mathrm{a} b}$ Mean values within a row with unlike superscript letters were significantly different $(P<0.05)$.

${ }^{*}$ Rats were fed the basal diet (HMB group), the diet containing Camembert (HMCp group) or fermented milk (HMfm group) for 6 weeks; for details of diets and procedures, see Table 1 and p. 430 .

(HMB), significantly and similarly increased with both dairy products. However, when expressed as $\mu \mathrm{g}$ bile acids/g faeces, this increase remained significant in HMfm rats only. HMCp rats was intermediate between HMB and HMfm rats. $\omega$-Muricholic acid, whether expressed as \% composition or faecal concentration, was also significantly increased in HMfm rats. Ursocholic acid, bacterial epimer of cholic acid, was not detected in the HMB rats, and occasionally appeared in faeces of rats fed either dairy product, but the increase did not reach statistical significance. Neither dairy product affected the other secondary bile acids, deoxycholic and lithocholic acids, resulting from $7 \alpha$-dehydroxylation of cholic and chenodeoxycholic respectively. Last, total bile acid concentration in faeces was the lowest in HMB rats and the highest in the HMfm rats. It was intermediate in the
HMCp rats, and did not significantly differ from both other groups.

\section{Discussion}

The soft cheese Camembert contained a complex microbiota mainly composed of Streptococcus thermophilus, Lactobacillus, Lactococcus, Leuconostoc, Hafnia alvei and Geotrichum, each reaching at least 7-8 log colony forming units/g. Our present approach is the first experimental study on the impact of cheese consumption on the intestinal microbiota. The objectives were: first, to determine the capacity of the cheese micro-organisms to survive through the gastrointestinal transit; second, to study the influence of these bacteria on a human microbiota established in our rat model; third, to study the influence of

Table 6. Distribution and concentration of bile acids in faeces from human microbiota-associated rats* (Mean values with their standard errors for five rats per group)

\begin{tabular}{|c|c|c|c|c|c|c|}
\hline \multirow[t]{2}{*}{ Group... } & \multicolumn{2}{|c|}{ HMB } & \multicolumn{2}{|c|}{ HMCp } & \multicolumn{2}{|c|}{ HMfm } \\
\hline & Mean & SEM & Mean & SEM & Mean & SEM \\
\hline \multicolumn{7}{|l|}{ Bile acids (mass \%) } \\
\hline Cholic & $8 \cdot 86$ & 0.62 & $7 \cdot 42$ & 0.48 & $7 \cdot 19$ & 0.37 \\
\hline Deoxycholic & $43 \cdot 10$ & 3.02 & $42 \cdot 35$ & $1 \cdot 12$ & $40 \cdot 06$ & $1 \cdot 33$ \\
\hline Ursocholic & 0.00 & 0.00 & 0.89 & 0.89 & 1.69 & 1.04 \\
\hline Chenodeoxycholic & $17 \cdot 15$ & 8.83 & $5 \cdot 10$ & 0.76 & 5.44 & 0.23 \\
\hline Lithocholic & $13 \cdot 57$ & $5 \cdot 54$ & $16 \cdot 74$ & $1 \cdot 60$ & $11 \cdot 32$ & $1 \cdot 36$ \\
\hline Ursodeoxcholic & $0.00^{\mathrm{a}}$ & 0.00 & $3.08^{b}$ & 1.33 & $3 \cdot 74^{\mathrm{b}}$ & 0.39 \\
\hline$\beta$-Muricholic & $9 \cdot 62$ & $2 \cdot 58$ & $13 \cdot 10$ & 1.84 & $13 \cdot 69$ & 0.54 \\
\hline$\omega$-Muricholic & $7 \cdot 70^{\mathrm{a}}$ & 2.49 & $11 \cdot 32^{\mathrm{a}}$ & 1.05 & $16 \cdot 88^{\mathrm{b}}$ & 1.06 \\
\hline \multicolumn{7}{|c|}{ Bile acids ( $\mu \mathrm{g} / \mathrm{g}$ faeces) } \\
\hline Cholic & 0.86 & 0.19 & 0.85 & 0.05 & $1 \cdot 32$ & 0.17 \\
\hline Deoxycholic & $4 \cdot 34$ & $1 \cdot 25$ & 5.03 & 0.76 & 7.59 & $1 \cdot 39$ \\
\hline Ursocholic & 0.00 & 0.00 & 0.10 & 0.10 & 0.30 & 0.18 \\
\hline Chenodeoxycholic & $1 \cdot 36$ & 0.52 & 0.59 & 0.09 & 1.04 & $0 \cdot 20$ \\
\hline Lithocholic & $1 \cdot 10$ & 0.45 & 1.87 & 0.02 & 1.96 & 0.07 \\
\hline Ursodeoxcholic & $0.00^{\mathrm{a}}$ & 0.00 & $0.38^{a b}$ & 0.18 & $0.69^{b}$ & 0.14 \\
\hline$\beta$-Muricholic & 1.01 & 0.35 & 1.66 & 0.51 & $2 \cdot 62$ & 0.54 \\
\hline$\omega$-Muricholic & $0.91^{a}$ & 0.46 & $1 \cdot 34^{a}$ & 0.21 & $3 \cdot 26^{b}$ & 0.68 \\
\hline Total bile acids & $9 \cdot 58^{a}$ & 1.93 & $11 \cdot 82^{\mathrm{ab}}$ & 1.64 & $18 \cdot 78^{\mathrm{b}}$ & 3.06 \\
\hline
\end{tabular}


cheese consumption on intestinal microbiota metabolism. The results were compared with those obtained with the fermented milk containing Streptococcus thermophilus, Lactobacillus delbrueckii bulgaricus and Lactobacillus casei strains. This fermented milk has been previously studied with our model (Djouzi et al. 1997).

It has been shown that when human faecal microbiota is inoculated into rats, the microbiota maintains its major metabolic characteristics (Andrieux et al. 1991; Rumney \& Rowland, 1992). However, little is known on the reproducibility of the microbial populations implanted in intestine of rats. PCR-TTGE analyses showed a high reproducibility and stability over time of the faecal microbiota profiles between groups in our present model. The HM rat model was therefore very useful to compare the effects of dietary components on the microbiota established in the intestinal ecosystem. Moreover, the similarity of faecal and caecal microbiota profiles suggested that the bacterial metabolism assessed in faecal samples could be related to metabolic variables analysed in caecal contents. SCFA were analysed in the caecum rather than in faeces because of their rapid absorption through the colonic epithelium (Macfarlane et al. 1991).

In HM rats fed the diet containing Camembert for several weeks, many microbial species from cheese were found at high levels in faecal samples, i.e. Streptococcus thermophilus, mesophilic Lactobacillus, yeast and Geotrichum. These micro-organisms were not found in rats fed the basal diet, demonstrating their capacity to resist during the gastrointestinal transit. The results obtained with the fermented milk confirmed the survival of Lactobacillus casei, but not that of Lactobacillus delbrueckii bulgaricus and Streptococcus thermophilus. Although lactic acid bacteria were found at high concentrations when culture analyses were used, they were not detected when using universal PCR primers and TTGE, suggesting that the transient bacteria constitute only a minor part of the total microbiota. Similarly, Tannock et al. (2000) did not detect Lactobacillus rhamnosus DR20 in human faecal samples using PCR-denaturing gradient gel electrophoresis and universal primers, whereas Lactobacillus rhamnosus was detected when group specific primers Lac1 and Lac2GC or culture analysis were used (Walter et al. 2001). In our present study, the use of the Lac1 and Lac 2GC primers lowered the detection limit so that Lactobacilli could be detected by PCR-TTGE in faecal samples in HMfm and HMCp groups during consumption of the dairy products. Lactobacillus species could be detected even in the presence of DNA from the predominant members of the faecal microbiota (about $10^{11}$ cells/ g) in the PCR. With the same approach, using specific primers, Satokari et al. (2001) detected Bifidobacterium lactis $\mathrm{Bb} 12$ in human subjects during the ingestion of Bb12 in fermented milk.

Bacterial PCR-TTGE detects 90-99\% of the numerically dominant bacterial species in faecal samples (Zoetendal et al. 1998). We observed only slight variations of the PCR-TTGE profiles during dairy product consumption, suggesting that the dominant microbiota was not greatly modified especially when rats were fed Camembert. This agrees with previous studies in gnotobiotic rats (Djouzi et al. 1997) and in human subjects, thus showing the stability of the dominant autochthonous faecal microbiota during consumption of fermented milks containing yoghurt starters, Lactobacillus casei or Bifidobacterium (Bartram et al. 1994; Guérin-Danan et al. 1998; Tannock et al. 2000; Satokari et al. 2001). However, with group-specific PCR primers we were able to monitor the variations of Lactobacilli at sub-dominant level.

It is difficult to prove that bacteria from cheese exert metabolic activity when they pass through the intestinal tract of HM rats. Caecal SCFA concentration was not significantly affected by cheese ingestion. However, several changes were observed in rats fed Camembert. The increase of mucolytic activities in HMCp rats may be related to the mucolytic activities of the cheese bacteria. The decrease in azoreductase activity suggests that the cheese bacteria, which do not produce reductase, were able to influence the reductase activity of the human microbiota. This beneficial influence of cheese was also observed with fermented milk. In addition, with fermented milk we observed a significant increase of $\beta$-galactosidase activity that could be due either to the $\beta$-galactosidase activity of bacteria provided by fermented milk or to the lactose contained in fermented milk used as substrate by the autochthonous microbiota.

Bile acid modifications in faeces are also a sensitive marker of microbial activity in the hindgut, since bile acid molecular profile and different bile acids exhibit distinct biological effects. To our knowledge, the effect of dairy products on bile acid profile in faeces has not been investigated before. We demonstrated an increase in bacterial epimerisation of bile acids under cheese and fermented milk consumption. This effect was more pronounced with fermented milk. As for other enzyme activities investigated in our present study, the question arises whether this was carried out by microbial enzymes from the dairy products or from the indigenous microbiota as modified by dairy product consumption. The first hypothesis might be the right one, since we also observed epimerisation of bile acids in germ-free rats fed Camembert (results not shown). Microbial epimerisation of bile acids is not direct, but proceeds in two steps catalysed by two hydroxysteroid dehydrogenases. Epimerisation of cholic and chenodeoxycholic acids to ursocholic and urosdeoxycholic acids respectively requires a $7 \alpha$ - and a $7 \beta$-hydroxysteroid dehydrogenase, whereas epimerisation of $\beta$-muricholic to $\omega$-muricholic acid requires a $6 \alpha$ - and a $6 \beta$-hydroxysteroid dehydrogenase. These enzymes would therefore be present in dairy products and would operate in the digestive tract of a HM rat model. This is of particular interest for health, since hydrophilic bile acids, such as ursodeoxycholic and muricholic acids, prevent cholestasis and liver damage (Milkiewicz et al. 2002). Ursodeoxycholic acid has been shown to be a chemopreventive agent (Earnest et al. 1994) and inhibits cell proliferation but does not induce apoptosis (Martinez et al. 1998). Interestingly, faecal deoxycholic and lithocholic acids, which are considered as tumour promoters in the colon (Cohen et al. 1980; Milovic et al. 2001), were not significantly affected by feeding on either dairy product, so that the overall faecal bile acid profile was displaced towards selective 
enrichment in beneficial bile acids. This of course should be further evaluated in human subjects.

The results obtained here show that, as fermented milk, Camembert consumption did not greatly modify the dominant intestinal microbiota or the major metabolic activities. But, we observed that micro-organisms from cheese microbiota were able to partly survive the intestinal transit. Moreover, cheese consumption led to a potentially beneficial influence on the intestinal metabolism such as a decrease in azoreductase activity, a decrease in $\mathrm{NH}_{3}$ concentration and an increase of the proportion of ursodeoxycholic.

Because great variability was observed between human subjects (Andrieux et al. 2002), we cannot generalise on the results obtained with a single microbiota. Nevertheless, several animal and human studies have observed the decrease of deleterious activities or metabolite production during probiotic administration (Bezkorovainy, 2001).

More information should be obtained in human volunteers to confirm our present results and identify the micro-organisms from cheese able to survive in the human intestinal tract and exert metabolic activities. Moreover, other investigations will be necessary to investigate mechanisms underlying potentially beneficial effects of cheese.

\section{Acknowledgements}

The authors thank the manufacturers Lactalis Beurre et Fromage for providing the Camembert (Camembert Président).

\section{References}

Andrieux C, Lory S, Dufour-Lescoat C, de Baynast R \& Szylit O (1991) Physiological effects of inulin in germ-free rats and in heteroxenic rats inoculated with a human flora. Food Hydrocolloid 5, 49-56.

Andrieux C, Membre JM, Cayuela C \& Antoine JM (2002) Metabolic characteristics of the fecal microflora in humans from three age groups. Scand J Gastroenterol 7, 792-798.

Bartram HP, Sheppach W, Gerlach S, Ruckdeschel G, Kelber E \& Kasper H (1994) Does yogurt enriched with Bifidobacterium longum affect colonic microbiology and fecal metabolism in healthy subjects? Am J Clin Nutr 59, 428-432.

Bezkorovainy A (2001) Probiotics: determinants of survival and growth in the gut. Am J Clin Nutr 73, 399S-405S

Blaut M, Collins MD, Welling GW, Doré J, van Loo J \& de Vos W (2002) Molecular biological methods for studying the gut microbiota: the EU human gut flora project. Br J Nutr 87, S203-S211.

Cohen BI, Raicht RF, Deschner EE, Takahashi M, Sarwal AN \& Fazzini E (1980) Effect of cholic acid feeeding on $N$-methyl- $N$ nitrosourea-induced colon tumors and cell kinetics in rats. $J$ Natl Cancer Inst 64, 573-578.

De Roos NM \& Katan MB (2000) Effects of probiotic bacteria on diarrhea, lipid metabolism, and carcinogenesis: a review of papers between 1988 and 1998. Am J Clin Nutr 71, 405-411.

Djouzi Z, Andrieux C, Degivry MC, Bouley C \& Szylit O (1997) The association of yogurt starters with Lactobacillus casei DN 114.001 in fermented milk alters the composition and metabolism of intestinal microflora in Germ-free rats and in human flora-associated rats. J Nutr 127, 2260-2266.

Drospy G \& Boy J (1961) Détermination de l'ammoniémie (méthode automatique par dialyse) (Determination of ammonia in the blood (automatic method using dialysis)). Ann Biol Clin (Paris) 19, 313-318.

Earnest DL, Holubec H, Wali RK, Jolley CS, Bissonette M, Bhattacharyya AK, Roy H, Khare S \& Brasitus TA (1994) Chemoprevention of azoxymethane-induced colonic carcinogenesis by supplemented dietary ursodeoxycholic acid. Cancer Res 54, 5071-5074.

Godon JJ, Zumstein E, Dabert P, Habouzit F \& Moletta R (1997) Diversity of microbial genes encoding small-subunit rRNA in an anerobic digestor. Appl Environ Microbiol 63, 2802-2813.

Goldin BR (1998) Health benefits of probiotics. Br J Nutr 80, S203-S207.

Guérin-Danan C, Chabanet C, Pedone C, Popot F, Vaissade P, Bouley C, Szylit O \& Andrieux C (1998) Milk fermented with yogurt cultures and Lactobacillus casei compared with yogurt and gelled milk: influence on intestinal microflora in healthy infants. Am J Clin Nutr 67, 111-117.

Heyman M \& Ménard S (2002) Probiotic microorganisms: how they affect intestinal pathophysiology. Cell Mol Life Sci 59, $1151-1165$.

Lenoir J, Lamberet G \& Schmidt JL (1995) L'élaboration d'un fromage: l'exemple du Camembert (Cheese making: example of Camembert). Pour la Science, Dossier hors série, March, pp. 64-73.

Lowry OH, Rosebrough NJ, Farr AL \& Randall RJ (1951) Protein measurement with the Folin-phenol reagent. J Biol Chem 193, $265-275$.

Macfarlane GT \& Cummings JH (1991) The colonic flora, fermentation and large bowel digestive function. In The Large Intestine: Physiology, Pathophysiology and Disease, pp. 51-92 [SF Phillips, JH Pemberton and RG Shorter, editors] New York: Raven Press.

Marteau Ph, Pochard Ph, Bouhnik Y \& Rambaud JC (1994) Survie et effets de lactobacilles acidophiles et bifidobactéries de produits laitiers fermentés dans le tube digestif de l'homme (Survival and effects of acidophilic lactobacilli and bifidobacteria from fermented milk products in the human digestive tract). Cah Nutr Diét 6, 336-340.

Marteau P, Seksik P \& Jian R (2002) Probiotics and intestinal health effects: a clinical perspective. Br J Nutr 88, S51-S57.

Martinez JD, Stratagoules ED, LaRue JM, Powell AA, Gause PR, Craven MT, Payne CM, Powell MB, Gerner EW \& Earnest DL (1998) Different bile acids exhibit distinct biological effects: the tumor promoter deoxycholic acid induces apoptosis and the chemopreventive agent ursodeoxycholic acid inhibits cell proliferation. Nutr Cancer 3, 111-118.

Milkiewicz P, Roma MG, Elias E \& Coleman R (2002) Hepatoprotection with tauroursodeoxycholate and betammuricholate against taurolithocholate induced cholestasis: involvement of signal transduction pathways. Gut 51, 113-119.

Milovic V, Teller IC, Murphy GM, Caspary WF \& Stein J (2001) Deoxycholic acid stimulates migration in colon cancer cells. Eur J Gastroenterol Hepatol 13, 945-949.

Reid G (1999) The scientific basis for probiotics strains of Lactobacillus. Appl Environ Microbiol 65, 3763-3768.

Rumney CJ \& Rowland IR (1992) In vivo and in vitro models of the human colonic flora. Crit Rev Food Sci Nutr 31, 299-331.

Satokari RM, Vaughan EE, Akkermans ADL, Saarela M \& De Vos WM (2001) Bifidobacterial diversity in human feces detected by genus-specific PCR and denaturating gradiant gel electrophoresis. Appl Environ Microbiol 67, 504-513.

Salminen S, Bouley C, Boutron-Ruault MC, Cummins JH, Franck A, Gibson GR, Isoluari E, Moreau MC, Roberfroid M \& Rowland I (1998) Functional food science and gastrointestinal physiology and function. Br J Nutr 80, S147-S171. 
Sanders ME (1993) Summary of conclusions from a consensus panel of experts on health attributes of lactic cultures: significance to fluid milk products containing cultures. J Dairy Sci 76, 1819-1828.

Seksik P, Rigottier-Gois L, Gramet G, Sutren M, Pochart P, Marteau P, Jian R \& Doré J (2003) Alterations of the dominant faecal bacterial groups in patients with Crohn's disease of the colon. Gut 52, 237-242.

Stanton C, Gardiner G, Lynch PB, Collins JK, Fitzgerald G \& Ross RP (1998) Probiotic cheese. Int Dairy J 8, 491-496.

Suau A, Bonnet R, Sutren M, Godon JJ, Gibson GR, Collins MD \& Doré J (1999) Direct analysis of genes endocoding 16S rRNA from complex communities reveals many novel molecular species within the human gut. Appl Environ Microbiol 65, 4799-4807.
Tannock GW, Munro K, Harmsen HJM, Welling GW, Smart J \& Gopal PK (2000) Analysis of the fecal microflora of human subjects consuming a probiotic product containing Lactobacillus rhamnosus DR20. Appl Environ Microbiol 66, 2578-2588.

Walter J, Hertel C, Tannock GW, Lis CM, Munro K \& Hammes WP (2001) Detection of Lactobacillus, Pediococcus, Leuconostoc, and Weissella species in human feces by using group-pecific PCR primers and denaturing gradient gel electrophoresis. Appl Environ Microbiol 67, 2578-2585. Zoetendal EG, Akkermans ADL \& De Vos WM (1998) Temperature gradient gel electrophoresis analysis of 16S rRNA from human fecal samples reveals stable and hosts specific communities of active bacteria. Appl Environ Microbiol 64, 3854-3859. 